\title{
Transposition
}

Musique et Sciences Sociales

Hors-série 1 | 2018

Musique, histoire, sociétés

\section{Le CRAL, « mi casa » (chez moi)}

\section{Marina Cañardo}

Traducteur : Elsa Broclain

\section{OpenEdition}

\section{Journals}

Édition électronique

URL : http://journals.openedition.org/transposition/1775

DOI : 10.4000/transposition. 1775

ISSN : 2110-6134

Éditeur

CRAL - Centre de recherche sur les arts et le langage

Référence électronique

Marina Cañardo, "Le CRAL, « mi casa » (chez moi) », Transposition [En ligne], Hors-série 1 | 2018, mis en ligne le 30 janvier 2018, consulté le 10 février 2020. URL : http://journals.openedition.org/ transposition/1775 ; DOI : 10.4000/transposition.1775

Ce document a été généré automatiquement le 10 février 2020.

La revue Transposition est mise à disposition selon les termes de la Licence Creative Commons Attribution - Partage dans les Mêmes Conditions 4.0 International. 


\title{
Le CRAL, « mi casa » (chez moi)
}

\author{
Marina Cañardo \\ Traduction : Elsa Broclain
}

1 Je m'appelle Marina Cañardo. J'ai fait mes études au département d'Arts (Musique) de l'université de Buenos Aires. J'ai ensuite soutenu et validé ma thèse dans cette université et à l'EHESS. Je suis Argentine et j'ai fait mon doctorat en cotutelle entre les deux institutions. Pendant mon doctorat et par la suite, j'ai effectué à diverses reprises le trajet aller-retour entre Buenos Aires et Paris. En 2007, j'ai réalisé mon premier séjour à Paris, et depuis lors, le CRAL (Centre de recherches sur les arts et le langage EHESS/CNRS : http://cral.ehess.fr/) est devenu «mi casa » (chez moi). Je n'oublierai jamais le temps que j'ai passé à travailler dans ses salles, ni les réunions aussi enrichissantes avec d'autres chercheurs, ni les heures passées devant ces ordinateurs communautaires dont le clavier me paraissait initialement aussi étrange que la langue française.

2 Je me souviens de mes collègues, devenus plus tard de chers amis, Sara Iglesias et Igor Contreras Zubillaga, organisant avec le CRAL le colloque «Le son des rouages » en 2007, sur les représentations musicales des rapports homme-machine au $\mathrm{xx}^{\mathrm{e}}$ siècle. Je me rappelle ma surprise devant la générosité et la confiance qu'ils ont démontrées à mon égard, lorsqu'ils m'ont confié la modération de l'une des tables rondes de ce colloque. Pour pouvoir le faire, ils avaient dû m'expliquer auparavant «les codes de l'étiquette universitaire française » (comme, par exemple, la présentation des participants de la manière suivante: "Vous avez étudié telle chose, vous avez publié telle autre...» comme si la personne en question ne savait pas qui elle était ou ce qu'elle avait publié!) qui m'ont semblé presque aussi bizarres que les touches «désordonnées» de leurs ordinateurs. J'ai beaucoup appris de cette expérience initiatique, et l'enthousiasme qu'elle a généré en moi a été à l'origine d'autres colloques, que j'ai par la suite moimême organisés avec d'autres collègues-amis du CRAL, comme celui que nous avons préparé conjointement avec le département de l'Audiovisuel de la Bibliothèque nationale de France le 21 octobre 2008 : « De la Phonothèque nationale à internet : 70 ans du Dépôt légal du disque ». 
3 Les séminaires de l'EHESS me reviennent également à l'esprit comme des moments d'apprentissage considérable. Les cours donnés par les "grands hommes» de l'institution, comme Luc Boltanski, Georges Didi-Huberman ou Roger Chartier furent particulièrement éclairants par leur manière de penser certains phénomènes sociaux. Plus spécifiquement, les séminaires portant sur des thèmes liés à la musique, de Denis Laborde («Faire la musique. Anthropologie de la création»), Esteban Buch (« Musique et politique au $\mathrm{xx}^{\mathrm{e}}$ siècle: histoire et théorie de la musique»), Rémy Campos (« Musiques “populaires”, 1820-1980») ou Laure Schnapper (« Analyse et histoire de la musique : théorie et pratique ») m'ont énormément apporté pour réfléchir à mes propres recherche doctorales. Celles-ci portaient sur la musique argentine enregistrée pendant la décennie 1920 (principalement le tango) et ses relations avec l'industrie du disque. Évidemment, aucun de ces séminaires n'abordait directement mon sujet, mais j'ai pu tirer de chacun d'eux quelque chose qui m'a permis de le repenser de manière plus globale, afin de comprendre le phénomène de la circulation des tangos à travers les disques comme une construction sociale de sens musical.

4 De manière générale, l'expérience de ces séminaires a déterminé pour toujours ma manière de faire de la recherche et d'enseigner. Dans chacune des activités que je mène depuis, je m'efforce de reproduire l'ouverture d'esprit et la pensée critique qui se dégageaient de ces rencontres. Je poursuis actuellement ma recherche sur des thèmes qui ont surgi comme des conséquences de mon doctorat: les liens entre la musique et l'industrie culturelle, l'interprétation musicale, la globalisation des genres musicaux et leurs réappropriations locales. Je suis professeure à l'université de Buenos Aires (où j'ai récemment gagné un concours en tant que Chef de TD), dans le doctorat de Musique de l'Université catholique argentine et professeure invitée dans d'autres universités argentines, comme l'Université nationale du Littoral ou l'Université nationale de Córdoba. La disposition même de mes élèves dans l'espace de la classe s'est transformée après mon passage par l'EHESS : on s'assoit maintenant autour d'une table-ronde, pour encourager l'échange des idées tel qu'il se produit à l'École.

5 Je conserve précieusement le souvenir du séminaire des doctorants du CRAL (devenu aujourd'hui le séminaire "La Recherche en Arts»), dont nous avons initié l'organisation en 2007, et qui n'a cessé de croître depuis. Un espace inestimable d'échange de connaissances et d'expériences, qui a surgi de l'initiative de doctorants de ce laboratoire et s'est étendu à d'autres étudiants. Ce type d'espace, permis par le CRAL, constitue un apport fondamental pour la formation des nouvelles générations de chercheurs. Les groupes de recherche qui se forment, à l'intérieur de ce laboratoire, avec des chercheurs d'origines diverses sont aussi particulièrement fructueux. J'ai eu la chance de participer à l'un d'entre eux entre 2009 et 2012 : «GLOBAMUS. Création musicale, circulation et marché d'identités en contexte global » dirigé par Emmanuelle Olivier (Résolution $\mathrm{N}^{\circ}$ ANR-08-CREA-067, Centre National de Recherche, http:// globalmus.net/).

6 En tant que membre de l'équipe GLOBAMUS, j'ai beaucoup appris sur la question du lien entre musiques et constructions identitaires dans des contextes très différents, mais avec des points communs particulièrement pertinents. Au sein de l'« équipe tango ", composée de chercheurs français et argentins spécialistes du genre, nous avons pu partager nos opinions autour de ce phénomène culturel complexe définit poétiquement par Enrique Santos Discépolo comme « un sentiment triste qui se danse ». Les échanges 
se sont élargis au-delà du groupe, et prirent la forme de colloques sur le tango, dont j'ai participé à l'organisation : deux à Buenos Aires (2010 et 2013) et un à Paris (2011).

7 La rencontre de 2011, la plus importante, a rassemblé des chercheurs, des musiciens, des poètes, des danseurs et des passionnés du tango en général, qui sont arrivés à Paris depuis les quatre coins du monde. Ce fut une expérience inoubliable pour tous ceux qui $\mathrm{y}$ participèrent et ne manquèrent ni un concert ni une milonga. Le tango $\mathrm{y}$ a été représenté "Comme il faut», selon le tango argentin au titre français, composé par Eduardo Arolas et rendu célèbre par la version de l'Orchestre Typique de Carlos Di Sarli. Ce fut l'une des organisations de colloque les plus prenantes et les plus divertissantes dont je me souvienne, grâce à une forte dose d'humour et la bonne disposition de Sophie Jacotot et Esteban Buch.

8 Je dédie ce paragraphe-souvenir final à Esteban Buch. En plus d'avoir suivi plusieurs de ses séminaires, c'est lui qui m'a introduite au CRAL, et il a su être un directeur de thèse généreux, devenant par la suite un collègue et ami. Je me souviens que lorsque nous discutions de ma soutenance et du pot qui suivrait, je lui avais demandé si nous pourrions faire cette " célébration" au CRAL parce que je sentais que c'était "chez moi ». Esteban, sans masquer la fierté que lui procurait cette demande, me répondit qu'aucun pot de thèse n'avait été fait là-bas, mais qu'il y avait un début à tout. Quelques jours plus tard, nous trinquions à mon doctorat dans «ma maison». Je porte aujourd'hui un toast pour davantage de doctorats, davantage de colloques, davantage d'échanges entre collègues-amis et, surtout, une longue vie à ce Centre qui continuera d'être le « chez » de beaucoup d'entre nous.

\section{AUTEURS}

\section{MARINA CAÑARDO}

Marina Cañardo est titulaire d'un doctorat en « Musique et musicologie du xx siècle » à l'École des hautes études en sciences sociales (EHESS) et d'un doctorat en « Histoire et théorie des arts » à l'université de Buenos Aires (UBA). Elle a étudié la flûte traversière, la composition et a travaillé dans des émissions radiophoniques de divulgation musicale. Elle participe régulièrement à des congrès nationaux et internationaux en Argentine, en France, au Japon, au Pérou et aux ÉtatsUnis. Ses travaux ont été publiés par des revues prestigieuses d'Amérique et d'Europe. Elle enseigne à l'université (UBA, Universidad Nacional del Litoral, Universidad Católica Argentina, Universidad Nacional de Córdoba) et fait partie de plusieurs groupes de recherche (UBA, EHESS, Universidad de La Plata, Universidad Torcuato Di Tella). Son livre Fábricas de músicas. Comienzos de la industria discográfica en la Argentina (1919-1930) a été publié en 2017 par le Gourmet Musical Ediciones (https://www.facebook.com/fabricasdemusicas/). Elle est actuellement directrice du Musée Carlos Gardel (Buenos Aires). 